\title{
Clinical Study \\ Digital Breast Tomosynthesis Changes Management in Patients Seen at a Tertiary Care Breast Center
}

\author{
L. Margolies, ${ }^{1}$ A. Cohen, ${ }^{2}$ E. Sonnenblick, ${ }^{1}$ J. Mandeli, ${ }^{3}$ P. H. Schmidt, ${ }^{2}$ J. Szabo, ${ }^{1}$ N. Patel, ${ }^{1}$ \\ G. Hermann, ${ }^{1}$ C. Weltz, ${ }^{2}$ and E. Port ${ }^{2}$ \\ ${ }^{1}$ Department of Radiology, Icahn School of Medicine at Mount Sinai, One Gustave Levy Place, P.O. Box 1234, New York, \\ NY 10029, USA \\ ${ }^{2}$ Department of Surgery, Icahn School of Medicine at Mount Sinai, One Gustave Levy Place, P.O. Box 1148, New York, NY 10029, USA \\ ${ }^{3}$ Department of Preventive Medicine, Icahn School of Medicine at Mount Sinai, 17 East 102 Street, New York, NY 10029, USA
}

Correspondence should be addressed to L. Margolies; laurie.margolies@mountsinai.org

Received 14 November 2013; Accepted 24 December 2013; Published 3 March 2014

Academic Editors: G. Borasi, J.-H. Chen, and M. D. Noseworthy

Copyright (C) 2014 L. Margolies et al. This is an open access article distributed under the Creative Commons Attribution License, which permits unrestricted use, distribution, and reproduction in any medium, provided the original work is properly cited.

Objectives. To study factors that predict changes in management with digital breast tomosynthesis (DBT). Methods. The Institutional Review Board approved this HIPAA compliant study. 996 patients had DBT with full field digital mammography (FFDM). Univariate analysis evaluated predictors of management change and cancer detection. Results. DBT changed management in 109 of 996 (11\%); 77 (71\%) required less imaging. Recalled patients after abnormal FFDM screen were most likely to have management change-25\% (24 of 97 patients) compared to $8 \%(13 / 163)$ of symptomatic patients and $10 \%(72 / 736)$ of screening patients $(P<$ $0.001)$. Dense breasted patients had a higher likelihood of having DBT change management: 13\% (68/526) compared to 9\% (41/470) $(P=0.03)$. Of the 996 patients, $19(2 \%)$ were diagnosed with breast cancer. 15 cancers (83\%) were seen on FFDM and DBT; 3 (17\%) were diagnosed after DBT $(0.3 \%, 95 \%$ CI: $0.1-0.9 \%)$. One recurrence was in the skin and was not seen on DBT nor was it seen on FFDM. The increase in cancer detection rate was $17 \%$ for asymptomatic patients, $0 \%$ for symptomatic patients, and $100 \%$ for recalled patients. Conclusions. DBT increased cancer detection rate by $20 \%$ and decreased the recall rate in $8-25 \%$. Advances in Knowledge. DBT led to a doubling of the cancer detection rate in recalled patients.

\section{Introduction}

Prospective trials of screening mammography demonstrate a reduction in breast cancer mortality $[1,2]$ yet concerns regarding false positive and false negative findings generate controversy [3]. In parallel with efforts to define populations who may benefit from supplemental ultrasound or MRI screening [4-8] technical innovations to improve mammography's sensitivity and specificity have included full field digital mammography (FFDM) $[9,10]$, computer aided detection (CAD) [11], and digital breast tomosynthesis (DBT) [12].

DBT images potentially reduce false positives caused by overlapping tissues which can mimic cancer while simultaneously reducing false negatives caused by tissue overlap which could obscure malignancy. Thus, DBT improves the sensitivity of screening mammography compared with FFDM while decreasing recall rates [13-15]. Much work investigating DBT, however, has been retrospective or performed on enriched data sets [16-18] and there are few studies on the role of DBT in populations at elevated risk for breast cancer. In a metaanalysis of 14 studies, no data was available regarding the clinical variables associated with enhanced cancer detection by DBT [13]. Here, we investigated groups of patients for whom DBT altered management. We sought to prospectively determine the effect on patient management of adding DBT to FFDM in asymptomatic, symptomatic, and recalled patients presenting to a dedicated breast imaging center and sought to identify clinical factors which could predict the added value of DBT.

\section{Methods and Materials}

This study was compliant with the Health Insurance Portability and Accountability Act (HIPAA) and was approved by 
the Institutional Review Board. A waiver of informed consent was granted.

From January 6, 2012, to November 28, 2012, all patients who presented or were referred to our comprehensive breast center for screening or diagnostic mammography were offered or randomly selected for the combined FFDM/DBT exam at no extra cost. During the prospective study period 1011 DBT examinations were performed; 15 patients were excluded due to recently diagnosed breast cancer, leaving 996 patients in the cohort.

The imaging was obtained and interpreted using standard clinical protocol including the use of CAD for FFDM (R2 Image Checker Version 9.3 Hologic Corporation, Bedford, MA). All images were obtained on a commercially available DBT Hologic Selenia Dimensions system (Hologic Corporation, Bedford, MA). All breasts were imaging in the craniocaudal (CC) and medial lateral oblique position (MLO). The FFDM and DBT images were obtained in the same compression using automatic exposure control; the $\mathrm{mA}, \mathrm{kVp}$, and dose were automatically selected by the mammography equipment. The DBT acquisition uses 15 projection images that were reconstructed into $1 \mathrm{~mm}$ thin sections for viewing on a Hologic workstation. Both FFDM and DBT images were viewed on the same Hologic workstations. One of four board certified MQSA certified radiologists with 3 to 10 years of FFDM experience and 8-20 months of DBT experience interpreted the images. Prior images and clinical history were made available.

A demographic, clinical data and result sheet were provided to the reading radiologist for each patient undergoing DBT. The radiologist prospectively recorded whether the patient presented for asymptomatic screening or symptomatic diagnostic evaluation or was recalled from an abnormal FFDM screening exam. Other clinical and demographic data including personal or family history of breast cancer, history of prior biopsy, and pathology results (if available) were recorded.

The radiologist first interpreted the FFDM images blinded to the DBT images and assigned one of the standard Breast Imaging Reporting and Database System (BI-RADS) [19] codes for breast density: fatty, scattered fibroglandular elements, heterogeneously dense or extremely dense. The same radiologist then assigned a BI-RADS assessment code and made recommendations for further evaluation if needed. The radiologist then accessed the DBT images, interpreted the DBT study, and recorded change in recommendations if any. Results were entered into a HIPAA compliant database.

Change in management was defined as a change in recommendation for additional imaging, biopsy, or followup interval. Cancer detection rates before (FFDM alone) and after the addition of DBT were assessed.

Univariate analysis was performed using a nonparametric chi-square (Pearson's chi-square) at the $\alpha=0.05$ level. Risk factors were tested as independent predictors of change in clinical management due to the addition of DBT. Proportions were compared using the $z$ ratio for difference between two independent proportions. Change in management was assigned per patient with no distinction made between unilateral or bilateral change. Cancer detection rates were calculated for FFDM and DBT. Change in cancer detection rate due to the addition of DBT was calculated. Statistical analysis was limited to a 95\% Confidence Interval due to lack of independence between subjects in the FFDM and DBT groups.

\section{Results}

The patient population presenting to our comprehensive breast cancer center was heterogeneous in terms of factors relevant to breast cancer risk but overall represented a cohort at increased risk. Of the 996 cases, 207 (21\%) had a history of breast cancer and $68(6.8 \%)$ had a mastectomy and underwent unilateral imaging. (see Tables $1(\mathrm{a})$ and $1(\mathrm{~b})$ ). A total of $711(71.4 \%)$ screening exams and $285(28.6 \%)$ diagnostic exams were performed in 996 patients. Screening exams were performed in $330(46.4 \%)$ women at normal risk, $188(26.4 \%)$ patients with a personal history of breast cancer, 185 (26.1\%) patients at increased risk for breast cancer due to a family history of breast cancer, a history of atypical lesions, and/or BRCA mutation, and $8(1.1 \%)$ patients with a distant history of breast intervention. A total of 285 (28.6\%) diagnostic exams were performed on 168 (58.7\%) patients presenting with symptoms, 97 (34.0\%) recalled for additional imaging, and $21(7.3 \%)$ presenting for short term followup (see Table 1(b)).

3.1. Impact of DBT on Management. For 887 of 996 (89\%) patients, results and recommendations did not change after DBT imaging, whereas DBT resulted in a change in management in 109 of 996 (11\%) patients (See Table 2). 79 of the 109 whose management was changed had bilateral imaging, and 30 had unilateral exams, due to previous contralateral mastectomy, short term followup for benign biopsy, and/or abnormal imaging, or because recall was only indicated for one side. Change in management affected 1 breast in 105 patients and both breasts in 4 patients. Of the 4 patients with bilateral changes, 2 patients had additional studies requested for one side, and fewer studies requested on the other side. The other 2 patients did not need recall after DBT was reviewed.

Of the 109 patients who had changes in management, 77 (71\%) did not need to be recalled for a diagnostic evaluation after DBT. Thus 77 of 996 (7\%) of all patients had a change in management requiring less additional imaging. For these 77 patients, 91 exams that would have been performed based on the FFDM were cancelled after the DBT exam was reviewed. The 91 cancelled exams included additional mammographic images in 53 patients, ultrasound in 10 patients, and both additional views and ultrasound in 14 patients (28 exams). In this group DBT served as the additional imaging which was included in the screening exam and, thus, the patient was not recalled for diagnostic imaging.

The 77 patients who would have been recalled had it not been for DBT were followed for an average of 17 months (range: 13-22 months). Upon review of their electronic medical record, no cancers have occurred in these 77 women. 48 did have normal imaging subsequent to the index exam. 29 have not returned for a followup imaging exam to date. 
Table 1: (a) Patient demographics. (b) Patient demographics by exam type.

(a)

\begin{tabular}{lc}
\hline Number of patients & $n=996$ \\
\hline Age & \\
(i) Mean & 53.1 \\
(ii) Range & $25-87$ \\
Breast density & \\
(i) Fatty & $84 / 996(8.4 \%)$ \\
(ii) Scattered & $387 / 996(38.9 \%)$ \\
(iii) Heterogeneously dense & $454 / 996(45.6 \%)$ \\
(iii) Extremely dense & $71 / 996(7.1 \%)$ \\
Risk factors & \\
Personal history of breast cancer & $207 / 996(20.8 \%)$ \\
(i) Unilateral breast cancer & $198 / 207(95.7 \%)$ \\
(ii) Bilateral breast cancer & $9 / 207(4.3 \%)$ \\
(iii) Breast conserving therapy & $142 / 207(68.6 \%)$ \\
(iiii)Mastectomy ${ }^{*}$ & $68 / 207(32.9 \%)$ \\
$\quad{ }^{*} 3 / 6$ bilateral breast & \\
$\quad$ cancer patients had both & \\
$\quad$ BCT and mastectomy & \\
BRCA positivity & \\
ADH & $11 / 996(1.1 \%)$ \\
LCIS & $11 / 996(1.1 \%)$ \\
Nonspecified benign biopsy & $9 / 996(0.9 \%)$ \\
Family history of breast cancer & $368 / 996(36.9 \%)$ \\
(i) First degree & $324 / 996(32.5 \%)$ \\
(ii) Second degree & $183 / 324(56.5 \%)$ \\
(iii) Both & \\
(iiii) Not reported & \\
\hline
\end{tabular}

*3/9 bilateral breast cancer patients had a unilateral mastectomy and contralateral breast conserving therapy.

(b)

\begin{tabular}{lc}
\hline Number of patients & $n=996$ \\
\hline Screening exam & $n=711(71.4 \%)$ \\
(i) Unremarkable breast history & $330 / 711(46.4 \%)$ \\
(ii) Personal h/o breast cancer & $188 / 711(26.4 \%)$ \\
(iii) High risk & $185 / 711(26.1 \%)$ \\
(iiii) Distant h/o breast intervention & $8 / 711(1.1 \%)$ \\
Diagnostic exam & $n=285(28.6 \%)$ \\
(i) Symptoms & $167 / 285(58.6 \%)$ \\
(ii) Recalled for additional imaging & $97 / 285(34.0 \%)$ \\
(iii) Short term followup & $21 / 285(7.4 \%)$ \\
\hline
\end{tabular}

One patient presented to dermatology 2 months after the mammogram with a $3 \mathrm{~mm}$ cutaneous metastatic deposit. This was not seen in retrospect on FFDM or DBT nor was cancer found in breast tissue. No other patients have been diagnosed with breast cancer; that is, to date no false negatives have resulted from the changes in management based on DBT.

Thirty-four of the 109 (31\%) patients had a change in management where additional imaging was needed after
DBT. Thus 34 of 996 (3\%) patients had a change in management triggered by DBT requiring additional imaging. One of these patients was recommended to have short term followup imaging which had not been recommended after FFDM. The remaining 33 patients had 36 additional examinations performed after the addition of DBT. Of the 33 patients, 6 required additional mammogram images, 18 required ultrasound, and 6 patients required both additional mammogram images and ultrasound (12 exams). Biopsies were recommended in 5 patients after DBT alone or based on additional imaging prompted by DBT.

3.2. Factors Associated with Management Change. The 996 patients were categorized based on indication for examination as asymptomatic screening symptomatic patients presenting for diagnostic evaluation, or patients recalled from prior screening. Patients who were recalled from screening were significantly more likely to have a management change with the addition of DBT; 24 of the 97 patients (25\%) who had DBT as part of recall had a change in management, compared to 14 of the 167 symptomatic patients $(8 \%)$ and 71 of the 732 asymptomatic patients $(10 \%)(P<0.001)$. There was no difference in impact of DBT in the screening and symptomatic diagnostic groups; 71 of the 732 patients $(10 \%)$ who were asymptomatic had a change in management compared to 14 of the 167 symptomatic patients $(8 \%)$ who had a change in management $(P=0.12,0.19$, resp.).

Breast density was evaluated at the time of FFDM. Patients with BI-RADS types 3 and 4 breast density had a significantly higher likelihood of having DBT change management: 68 of 525 (13\%) with heterogeneously or extremely dense breasts had a change recommended compared to 41 of $471(9 \%)$ of those with fatty or scattered fibroglandular breast tissue $(P=0.03)$.

Patient age was not associated with an increased likelihood of change in management. For women younger than 50 years old, 52 of $412(13 \%)$ had a change in management recommended, compared to 57 of $584(10 \%)$ for those 50 and older $(P=0.15)$. For women under 50 the most common change in management was reduction in need for additional imaging in 39 of 52 cases (75\%).

Although the subgroups are too small to allow for statistically significant data, there was no trend identified associated with identifiable risk factors for breast cancer, such as personal history, family history, prior diagnosis of high risk lesion including atypia or LCIS, or BRCA mutation, that were associated with a change in management based on DBT.

3.3. Cancer Detection. Of the 996 patients, 19 (2\%) were diagnosed with breast cancer (See Table 4). 15 of 18 cancers $(83 \%)$ were seen on both FFDM and DBT, while 3 of the $18(17 \%)$ were diagnosed on DBT findings alone or after additional imaging generated by DBT $(0.3 \%$, 95\%CI: $0.1-$ $0.9 \%$ ) (see Table 3 ). One cancer was a cutaneous metastatic deposit that was seen neither on FFDM or DBT. Of the 3 patients with cancer detected by DBT, 2 had biopsies based on DBT results alone, while 1 patient had additional imaging triggered by DBT findings and US confirmed a suspicious lesion which was biopsied under US guidance. 
TABLE 2: Factors affecting change in management.

\begin{tabular}{|c|c|c|c|}
\hline Factors & Change in management $n=109$ & No change in management $n=887$ & $P$ value \\
\hline \multicolumn{4}{|l|}{ Breast density } \\
\hline (i) Dense & $68 / 109(62.4 \%)$ & $457 / 887(51.5 \%)$ & \multirow{2}{*}{$P=0.034$} \\
\hline (ii) Not dense & $41 / 109(37.6 \%)$ & $430 / 887(48.5 \%)$ & \\
\hline \multicolumn{4}{|c|}{ Exam indications } \\
\hline (i) Screening & $72 / 109(66.1 \%)$ & $639 / 887(72.0 \%)$ & \multirow{2}{*}{$P<0.001$} \\
\hline (ii) Diagnostic & $37 / 109(33.9 \%)$ & $248 / 887(28.0 \%)$ & \\
\hline \multicolumn{4}{|c|}{ Prior diagnosis of Atypia or LCIS } \\
\hline (i) Yes & 2/109 (1.8\%) & $18 / 887(2.0 \%)$ & \multirow{2}{*}{$P=0.984$} \\
\hline (ii) No & $107 / 109(98.2 \%)$ & $869 / 887(98.0 \%)$ & \\
\hline \multicolumn{4}{|l|}{ BRCA mutation } \\
\hline (i) Yes & $1 / 109(0.9 \%)$ & $12 / 887(1.4 \%)$ & \multirow{2}{*}{$P=0.705$} \\
\hline (ii) No & 108/109 (99.1\%) & $875 / 887(98.4 \%)$ & \\
\hline \multicolumn{4}{|c|}{ Family history of breast cancer } \\
\hline (i) Yes & $40 / 109(36.7 \%)$ & 285/887 (32.1\%) & \multirow{2}{*}{$P=0.325$} \\
\hline (ii) No & $69 / 109(63.3 \%)$ & $602 / 887(67.9 \%)$ & \\
\hline \multicolumn{4}{|c|}{ Personal history of breast cancer } \\
\hline (i) Yes & $18 / 109(16.5 \%)$ & $189 / 887(21.3 \%)$ & \multirow{2}{*}{$P=0.244$} \\
\hline (ii) No & $91 / 109(83.5 \%)$ & 698/887 (78.7\%) & \\
\hline \multicolumn{4}{|l|}{ Age } \\
\hline (i) $<50$ & $52 / 109(47.7 \%)$ & $360 / 887(40.6 \%)$ & \multirow{2}{*}{$P=0.154$} \\
\hline (ii) $\geq 50$ & $57 / 109(52.3 \%)$ & $527 / 887(59.4 \%)$ & \\
\hline
\end{tabular}

Ninety-five biopsies were recommended in the 887 patients without a management change. 81 biopsies were performed with cancer detected in 16 of 81 (20\%). Of the 14 patients for whom biopsy was recommended but not performed, 8 were lost to followup, and 6 biopsies were cancelled after repeat imaging performed immediately preceding the biopsy led to cancellation of the recommendation. Of the 6 patients with biopsies cancelled at the time of the procedure, 2 went on to have biopsies after 6 month followup imaging was performed. Both resulted in benign findings.

The addition of DBT led to biopsies in 5 patients with $3(60 \%)$ demonstrating cancer. At surgery, tumor sizes were $1.4 \mathrm{~cm}, 1.6 \mathrm{~cm}$, and microinvasive (see Table 3 ).

With FFDM alone, the overall cancer detection rate was 15 of $996(1.5 \%)$. With the addition of DBT the cancer detection rate rose to 18 of $996(1.8 \%)$, an increase of $20 \%(0.3 \%, 95 \% \mathrm{CI}$ : $0.1-0.9 \%)$.

3.4. Factors Associated with Cancer Detection. Of the 15 patients whose cancers were detected by FFDM and DBT, 6 were seen in the 732 asymptomatic screened patients and thus the cancer detection rate was $1 \%$ or $9.6 / 1000$ in this screened population. This high rate of screen detected cancers is expected in this high risk population. Among the 167 symptomatic diagnostic patients, 7 cancers were detected; cancer detection rate was 7 of 167 (4\%) or 41.9/1000. Among those recalled from FFDM screening for diagnostic mammography, 2 of 97 (2\%) had cancer diagnosed. Of the 3 additional cancers detected by DBT, 1 was in an asymptomatic woman and 2 were in women who were recalled from screening FFDM. Thus the increase in cancer detection rate based on the addition of DBT was $17 \%$ for asymptomatic patients, $0 \%$ for symptomatic patients, and $100 \%$ for those who were recalled from screening.

Of the 15 cancers diagnosed by both FFDM and DBT, cancer detection rate was similar between those with dense breasts (7 of 525,1.3\%) and those with fatty or scattered fibroglandular breasts ( 8 of $471,1.7 \%$ ). The 3 additional cancers diagnosed by DBT alone were all detected in patients with dense breasts.

The 3 patients diagnosed with breast cancer by DBT did not differ significantly from the other 15 diagnosed when comparing patient age, or any other risk factor related to cancer development; however, both were very small groups.

\section{Discussion}

Screening mammography with DBT compared with FFDM alone decreases recall rates while improving cancer detection $[14,20,21]$. Others have investigated DBT in recalled patients and have shown increased reader accuracy $[14,22,23]$. The relative benefit of DBT in patients with dense breasts is of interest, as breast density is a risk factor for breast cancer $[24,25]$ and reduces mammographic sensitivity [26-28]. The added benefit of DBT in subpopulations of patients, such as those at increased risk for breast cancer or younger women explored in this study, has not been previously described. Furthermore, a large proportion of studies evaluating DBT involve only small numbers of patients, many with fewer than 250 subjects [13].

In a recent important study, constituting the largest DBT screening study to date of women between the ages of 50 
TABLE 3: Cancers diagnosed.

\begin{tabular}{|c|c|c|c|c|c|c|c|}
\hline Age & $\begin{array}{c}\text { Exam } \\
\text { indication }\end{array}$ & $\begin{array}{c}\text { Breast } \\
\text { density: } \\
\text { BI-RADS }\end{array}$ & $\begin{array}{c}\text { Cancer } \\
\text { detected by }\end{array}$ & $\begin{array}{l}\text { Type of } \\
\text { biopsy }\end{array}$ & Tumor type & $\begin{array}{l}\text { Type of } \\
\text { surgery }\end{array}$ & $\begin{array}{l}\text { Largest } \\
\text { tumor size on } \\
\text { excision } \\
(\mathrm{mm})\end{array}$ \\
\hline 69 & Screening & 3 & FFDM & Stereo & DCIS & Mast & DCIS \\
\hline 75 & Diagnostic & 2 & FFDM & US & IDC & Unk $^{*}$ & Unk $^{*}$ \\
\hline 55 & Screening & 3 & FFDM & US & DCIS & Unk $^{*}$ & Unk $^{*}$ \\
\hline 46 & Diagnostic & 2 & FFDM & US & IDC & BCT & 20 \\
\hline 49 & Diagnostic & 2 & FFDM & US & ILC & BCT & 11 \\
\hline 47 & Diagnostic & 2 & FFDM & US & IDC & BCT & 5 \\
\hline 57 & Screening & 3 & FFDM & Punch bx & IDC & Mast & 3 \\
\hline 56 & Screening & 3 & FFDM & US & ILC/IDC & Mast & 18 \\
\hline 50 & Diagnostic & 2 & FFDM & US & IDC & BCT & 14 \\
\hline 56 & Diagnostic & 3 & FFDM & US & DCIS & BCT & 7 \\
\hline 47 & Screening & 2 & FFDM & Stereo & DCIS & Mast & DCIS \\
\hline 46 & Screening & 4 & FFDM & US & IDC & Mast & 11 \\
\hline 69 & Screening & 2 & FFDM & US & IDC & BCT & 4 \\
\hline 53 & Diagnostic & 3 & FFDM & MRI & IDC & Mast & $\mathrm{Unk}^{*}$ \\
\hline 49 & Diagnostic & 1 & FFDM & US & IDC & Neoadj & - \\
\hline 42 & Diagnostic & 3 & FFDM & US & IDC & Mast & 18 \\
\hline 61 & Screening & 4 & DBT & US & IDC/ILC & BCT & 14 \\
\hline 66 & Diagnostic & 3 & DBT & US & IDC & BCT & DCIS \\
\hline 59 & Diagnostic & 3 & DBT & US & $\begin{array}{c}\text { IDC/lobular } \\
\text { features }\end{array}$ & BCT & 16 \\
\hline
\end{tabular}

${ }^{*}$ Surgery performed at outside institution, unknown surgical pathology. LEGEND:

Asymp: asymptomatic

Symp: symptomatic

BI-RADS breast density categories:

BI-RADS 1 fatty breasts

BI-RADS 2 scattered fibroglandular elements

BI-RADS 3 heterogeneously dense

BI-RADS 4 extremely dense

IDC: invasive ductal cancer

ILC: invasive lobular cancer

BCT: breast conservation therapy

Unk: unknown.

and 69 , Skaane et al. found a $15 \%$ decrease in recall rate using DBT in 12,631 women, and a $27 \%$ improvement in the cancer detection rate (from 6.1/1000 to 8/1000) [20]. While results of this study of biennial screening showed that DBT contributed significantly to increased mammographic accuracy, this trial may underestimate the added benefit of DBT in the USA where many women begin annual screening at age 40 . In our study, $10 \%$ of asymptomatic screening patients had a change in management based on DBT, and the cancer detection rate increased $14 \%$. We did not, however, find a significantly higher cancer detection rate for younger women $(<50)$ based on DBT findings. In the context of diagnostic patients, DBT may replace spot compression views [14] but does not replace ultrasound. Although not included in this cohort of patients, we have seen cancers that are occult on DBT but visible on ultrasound. This is a known limitation of DBT; DBT is still mammography and shares some of the limitations of FFDM. Cancers can be invisible for many reasons on both DBT and
FFDM. Even on DBT some cancers will merge imperceptibly with the surrounding parenchyma and if there is not adjacent fat the cancer can be difficult if not impossible to detect. Other cancers that do not have calcifications or that do not distort the normal breast architecture can be missed on FFDM as well as DBT [29].

While improved cancer detection is an important endpoint in assessing the added benefit of DBT, the reduction in false positives and recall rate is also significant. In 2009, the United States Preventive Services Task Force (USPSTF) reversed their long standing recommendation for yearly mammography in women aged 40-49 largely due to the harms related to false positives and "unnecessary" biopsies [3]. Our findings demonstrated a 9.5\% reduction in need for additional imaging among all screened and diagnostic patients and a $25 \%$ reduction in recalled patients. $13 \%$ of women under age 50 had a change in management, and $75 \%$ of these changes resulted in fewer imaging studies performed. 
TABLE 4: (a) Cancers diagnosed on DBT. (b) Imaging findings of diagnosed cancers.

(a)

\begin{tabular}{lccc}
\hline & Patient 19 & Patient 16 & Patient 1 \\
\hline Age & 61 & 59 & 66 \\
History of breast cancer & No & No & No \\
High risk & Yes & No & No \\
Risk factor & 2 family history & - & - \\
Symptoms & None & None (call back) & None (call back) \\
Imaging finding & Spiculated mass & Spiculated mass & Irregular mass \\
Breast density & Extremely dense & Heterogeneously dense & Heterogeneously dense \\
Type of biopsy & Ultrasound core & Ultrasound core & Ultrasound core \\
Focality & Unifocal & Unifocal & Unifocal \\
Nodal status & $1 / 1$ (0.1 cm) & $0 / 1$ & $0 / 1$ \\
Tumor size on excision & 14 mm & 16 mm & - (residual DCIS only) \\
Tumor type & IDC/ILC & IDC/lobular features & IDC/DCIS \\
Grade & Poorly differentiated & Moderately differentiated & Well-moderately differentiated \\
Molecular subtype (ER/PR/Her2) & $+/+/-$ & $+/+/-$ & $+/+/-$ \\
\hline
\end{tabular}

(b)

\begin{tabular}{|c|c|c|c|c|c|c|c|c|c|c|}
\hline Patient & $\begin{array}{c}\text { Seen on } \\
\text { FFDM }\end{array}$ & $\begin{array}{c}\text { Seen on } \\
\text { DBT }\end{array}$ & $\begin{array}{l}\text { Visualized } \\
\text { on US }\end{array}$ & Mass & $\begin{array}{l}\text { Imaging size } \\
(\mathrm{mm})\end{array}$ & Shape & Margin & Calcs & Distribution & Character \\
\hline 1 & No & Yes & Yes & Yes & $6 \mathrm{~mm}$ & Irregular & Irregular & No & - & \\
\hline 2 & Yes & Yes & Not done & No & - & - & - & Yes & Group & $\begin{array}{c}\text { Linear } \\
\text { branching }\end{array}$ \\
\hline 3 & Yes & Yes & Yes & Yes & $40 \mathrm{~mm}$ & Irregular & Spiculated & No & - & \\
\hline 4 & Yes & Yes & Yes & Yes & $9 \mathrm{~mm}$ & Irregular & Indistinct & No & - & \\
\hline 5 & Yes & Yes & Yes & Yes & $53 \mathrm{~mm}$ & Oval & Irregular & No & - & \\
\hline 6 & Yes & Yes & Yes & $\begin{array}{l}\text { Architectural } \\
\text { distortion }\end{array}$ & $13 \mathrm{~mm}$ & Irregular & Irregular & Yes & Group & Punctate \\
\hline 7 & Yes & Yes & Yes & Yes $^{*}$ & $10 \mathrm{~mm}$ & Irregular & Spiculated & No & - & \\
\hline $8^{* * * *}$ & No & No & No & No & - & - & - & & - & \\
\hline 9 & Yes & Yes & Yes & $\begin{array}{l}\text { Architectural } \\
\text { distortion }^{* *}\end{array}$ & $15 \mathrm{~mm}$ & Irregular & Spiculated & No & & \\
\hline 10 & Yes & Yes & Yes & Yes & $10 \mathrm{~mm}$ & Irregular ${ }^{* * *}$ & Irregular & No & & \\
\hline 11 & Yes & Yes & Yes & Yes* & $11 \mathrm{~mm}$ & $\begin{array}{l}\text { Round- } \\
\text { lobulated }\end{array}$ & $\mathrm{n} / \mathrm{a}$ & No & & \\
\hline 12 & Yes & Yes & Not Done & No & - & - & - & Yes & 2 Groups & $\begin{array}{c}\text { Pleo- } \\
\text { morphic }\end{array}$ \\
\hline 13 & Yes & Yes & Yes & Yes & $11 \mathrm{~mm}$ & $\begin{array}{l}\text { Round- } \\
\text { spiculated }\end{array}$ & $\mathrm{n} / \mathrm{a}$ & No & & \\
\hline 14 & Yes & Yes & Yes & Yes & $7 \mathrm{~mm}$ & Irregular & Spiculated & Yes & Associated & Coarse \\
\hline 15 & Yes & Yes & Yes & Yes & $3 \mathrm{~mm}$ & Oval & Lobulated & No & & \\
\hline 16 & No & Yes & Yes & Yes & $20 \mathrm{~mm}$ & Irregular & Spiculated & No & & \\
\hline 17 & Yes & Yes & Yes & Yes & $35 \mathrm{~mm}$ & Irregular & Lobulated & No & & \\
\hline 18 & Yes & Yes & Yes & Yes & $4 \mathrm{~mm}$ & Irregular & Spiculated & Yes & Segmental & $\begin{array}{l}\text { Hetero- } \\
\text { geneous }\end{array}$ \\
\hline 19 & No & Yes & Yes & Yes & $14 \mathrm{~mm}$ & Irregular & Spiculated & No & & \\
\hline
\end{tabular}

* Asymmetry seen on FFDM, mass on DBT only.

** DBT identified a second $8 \mathrm{~mm}$ mass with irregular margins.

*** Microlobulated mass on DBT.

${ }^{* * * *}$ Skin lesion was metastatic invasive ductal carcinoma. 
It is possible that DBT could lower false positives sufficiently to allow younger women to benefit from mammography with a reduction of the added harms that the USPSTF cautioned against.

The improved accuracy of DBT in those recalled from screening has been demonstrated by multiple authors [3033 ] and is confirmed in our study. Patients who were recalled from screening had the most significant increase in cancer detection rate compared to those who were classified as asymptomatic or symptomatic. Our results are similar to those of Skaane et al. [14] who reported that, of 84 women recalled from screening who were called "normal" after a standard mammogram workup, the addition of DBT found 2 additional cancers. This number is similar to ours where 2 of 97 women with cancer would have had a false negative result if only a standard mammogram workup was performed.

In patients at elevated risk for breast cancer due to family history, personal history of breast cancer, or prior biopsy (e.g., for atypia, LCIS, or multiple biopsies), we found no detectible incremental benefit for tomosynthesis compared to FFDM. In contrast, however, our data did indicate that DBT was most beneficial in patients with increased breast density, as the 3 additional cancers detected by DBT were found in patients with heterogeneously or extremely dense breasts. This data conflicts with that of Waldherr et al. who found the breast cancer detection rate to be about equally improved by DBT in those with fatty and dense breasts [30]. Recent legislation in some states has required patient notification of breast density and suggests that patients discuss possible supplemental screening $[34,35]$ with their healthcare providers. While we are not suggesting that DBT replaces ultrasound or MRI as supplemental screening, given our results DBT may benefit women with dense breasts.

The relative and added benefit of DBT in various subpopulations such as those at increased familial risk for breast cancer has not been previously described. The population studied here represents an example of such a cohort; $32 \%$ of patients in our cohort had at least 1 first degree relative with breast cancer, significantly higher than the $7.3 \%$ prevalence in the general population [36]. While supplemental MRI screening is established for screening of the highest risk cohorts, optimal screening regimens remain to be established for cohorts at intermediate risk. Our results show a change in management in $10 \%$ of asymptomatic women. And while there are no specific risk factors identified that predicted higher likelihood of DBT affecting cancer detection rate or lowering false positive findings in this group at elevated risk, the subgroups analyzed may be too small to demonstrate a benefit. As DBT is more widely evaluated, analysis of its benefits in specific groups of patients based on risk factors, such as those who are BRCA-positive, those with atypia or LCIS, and those who have undergone multiple previous biopsies, will be possible.

Overall, we found that DBT increased cancer detection rate by $19 \%$ and decreased the need for recall in $8-25 \%$, depending on the indication for DBT.

While our study was prospective and one of the larger series to date, it is nevertheless limited by sample size, and the heterogeneous and self selected nature of the population studied which may make the findings of this study difficult to generalize to the screening or callback settings. Our study population is that of a comprehensive breast center, not a screening center nor a retrospective, enriched reader study. The results therefore, while adding to the current literature, may be difficult to extrapolate. Ultrasound and additional mammographic views were readily available and the radiologists evaluating the FFDM knew that they would be seeing the DBT images. These factors may have impacted management recommendations and readings. However, the ready availability of multimodality imaging led to potentially interesting findings. For example, all the cancers diagnosed by DBT were detected by ultrasound to direct biopsy. This suggests that the incremental benefit of DBT compared to ultrasound screening may be small. Future studies to determine the incremental benefit of DBT compared to FFDM combined with screening ultrasound are needed to resolve this question.

In summary, in this study of the effect of DBT on management in a heterogeneous population, call back rates were reduced while cancer detection was increased, particularly in those with dense breasts. These results are consistent with similar studies in screening and recalled populations but are among the first data available highlighting DBT's impact on clinical management of patients at elevated risk for breast cancer seen in the setting of a comprehensive breast center. Future studies will add to the growing body of knowledge regarding the role of this technology in breast cancer screening and diagnosis and its optimal application.

\section{Conflict of Interests}

The authors declare that there is no conflict of interests regarding the publication of this paper.

\section{Acknowledgment}

The authors thank Barnaby Nicolas for his assistance with paper preparation.

\section{References}

[1] R. A. Smith, S. W. Duffy, and L. Tabar, "Breast cancer screening: the evolving evidence," Oncology, vol. 26, no. 5, pp. 471-486, 2012.

[2] L. Tabár, B. Vitak, T. H.-H. Chen et al., "Swedish two-county trial: impact of mammographic screening on breast cancer mortality during 3 decades," Radiology, vol. 260, no. 3, pp. 658-663, 2011.

[3] US Preventive Services Task Force, "Screening for breast cancer: US Preventive Services Task Force recommendation statement," Annals of Internal Medicine, vol. 151, no. 10, pp. 716-726, 2009.

[4] W. A. Berg, J. D. Blume, J. B. Cormack et al., "Combined screening with ultrasound and mammography vs. Mammography alone in women at elevated risk of breast cancer," The Journal of the American Medical Association, vol. 299, no. 18, pp. 21512163, 2008.

[5] W. A. Berg, Z. Zhang, D. Lehrer et al., "Detection of breast cancer with addition of annual screening ultrasound or a single 
screening MRI to mammography in women with elevated breast cancer risk," The Journal of the American Medical Association, vol. 307, no. 13, pp. 1394-1404, 2012.

[6] D. Saslow, C. Boetes, W. Burke et al., "American Cancer Society guidelines for breast screening with MRI as an adjunct to mammography," CA Cancer Journal for Clinicians, vol. 57, no. 2, pp. 75-89, 2007.

[7] H.R. 3102-112th Congress: Breast Density and Mammography Reporting Act of 2011, http://www.govtrack.us/congress/bills/ 112/hr3102.

[8] An Act to Add and Repeal Section 123222.3 of the Health and Safety Code, Relating to Mammograms, California State Senate, 11th Senate District, 2012.

[9] E. D. Pisano, R. E. Hendrick, M. J. Yaffe et al., "Diagnostic accuracy of digital versus film mammography: exploratory analysis of selected population subgroups in DMIST," Radiology, vol. 246, no. 2, pp. 376-383, 2008.

[10] E. D. Pisano, C. Gatsonis, E. Hendrick et al., "Diagnostic performance of digital versus film mammography for breastcancer screening," The New England Journal of Medicine, vol. 353, no. 17, pp. 1773-1783, 2005.

[11] R. F. Brem, J. Baum, M. Lechner et al., "Improvement in sensitivity of screening mammography with computer-aided detection: a multiinstitutional trial," American Journal of Roentgenology, vol. 181, no. 3, pp. 687-693, 2003.

[12] L. T. Niklason, B. T. Christian, L. E. Niklason et al., "Digital tomosynthesis in breast imaging," Radiology, vol. 205, no. 2, pp. 399406, 1997.

[13] N. Houssami and P. Skaane, "Overview of the evidence on digital breast tomosynthesis in breast cancer detection," The Breast, vol. 22, no. 2, pp. 101-108, 2013.

[14] P. Skaane, R. Gullien, H. Bjorndal et al., "Digital breast tomosynthesis (DBT): initial experience in a clinical setting," Acta Radiologica, vol. 53, no. 5, pp. 524-529, 2012.

[15] E. A. Rafferty, J. M. Park, L. E. Philpotts et al., "Assessing radiologist performance using combined digital mammography and breast tomosynthesis compared with digital mammography alone: results of a multicenter, multireader trial," Radiology, vol. 266, no. 1, pp. 104-113, 2013.

[16] M. G. Wallis, E. Moa, F. Zanca, K. Leifland, and M. Danielsson, "Two-view and single-view tomosynthesis versus full-field digital mammography: high-resolution X-ray imaging observer study," Radiology, vol. 262, no. 3, pp. 788-796, 2012.

[17] M. Noroozian, L. Hadjiiski, S. Rahnama-Moghadam et al., "Digital breast tomosynthesis is comparable to mammographic spot views for mass characterization," Radiology, vol. 262, no. 1, pp. 61-68, 2012.

[18] D. Gur, M. L. Zuley, M. I. Anello et al., "Dose reduction in digital breast tomosynthesis (DBT) screening using synthetically reconstructed projection images: an observer performance study," Academic Radiology, vol. 19, no. 2, pp. 166-171, 2012.

[19] C. J. D’Orsi, L. W. Bassett, W. A. Berg et al., "BI-RADS: mammography," in Breast Imaging Reporting and Data System: ACR BI-RADS-Breast Imaging Atlas, C. J. D'Orsi, E. B. Mendelson, and D. M. Ikeda, Eds., American College of Radiology, Reston, Va, USA, 4th edition, 2003.

[20] P. Skaane, A. I. Bandos, R. Gullien et al., "Comparison of digital mammography alone and digital mammography plus tomosynthesis in a population-based screening program," Radiology, vol. 267, no. 1, pp. 47-56, 2013.
[21] B. M. Haas, V. Kalra, J. Geisel, M. Raghu, M. Durand, and L. E. Philpotts, "Comparison of tomosynthesis plus digital mammography and digital mammography alone for breast cancer screening," Radiology, vol. 269, no. 3, pp. 694-700, 2013.

[22] A. Tagliafico, D. Astengo, F. Cavagnetto et al., "One-to-one comparison between digital spot compression view and digital breast tomosynthesis," European Radiology, vol. 22, no. 3, pp. 539544, 2012.

[23] S. P. Poplack, T. D. Tosteson, C. A. Kogel, and H. M. Nagy, "Digital breast tomosynthesis: initial experience in 98 women with abnormal digital screening mammography," American Journal of Roentgenology, vol. 189, no. 3, pp. 616-623, 2007.

[24] R. M. Tamimi, C. Byrne, G. A. Colditz, and S. E. Hankinson, "Endogenous hormone levels, mammographic density, and subsequent risk of breast cancer in postmenopausal women," Journal of the National Cancer Institute, vol. 99, no. 15, pp. 11781187, 2007.

[25] N. F. Boyd, L. J. Martin, J. M. Rommens et al., "Mammographic density: a heritable risk factor for breast cancer," Methods in Molecular Biology, vol. 472, pp. 343-360, 2009.

[26] M. T. Mandelson, N. Oestreicher, P. L. Porter et al., "Breast density as a predictor of mammographic detection: comparison of interval-and screen-detected cancers," Journal of the National Cancer Institute, vol. 92, no. 13, pp. 1081-1087, 2000.

[27] K. Kerlikowske, D. Grady, J. Barclay, E. A. Sickles, and V. Ernster, "Effect of age, breast density, and family history on the sensitivity of first screening mammography," The Journal of the American Medical Association, vol. 276, no. 1, pp. 33-38, 1996.

[28] N. F. Boyd, H. Guo, L. J. Martin et al., "Mammographic density and the risk and detection of breast cancer," The New England Journal of Medicine, vol. 356, no. 3, pp. 227-236, 2007.

[29] D. B. Kopans, "Digital breast tomosynthesis: a better mammogram," Radiology, vol. 267, no. 3, pp. 968-969, 2013.

[30] C. Waldherr, P. Cerny, H. J. Altermatt et al., "Value of one-view breast tomosynthesis versus two-view mammography in diagnostic workup of women with clinical signs and symptoms and in women recalled from screening," American Journal of Roentgenology, vol. 200, no. 1, pp. 226-231, 2013.

[31] M. J. Michell, A. Iqbal, R. K. Wasan et al., "A comparison of the accuracy of film-screen mammography, full-field digital mammography, and digital breast tomosynthesis," Clinical Radiology, vol. 67, no. 10, pp. 976-981, 2012.

[32] C. M. Hakim, D. M. Chough, M. A. Ganott, J. H. Sumkin, M. L. Zuley, and D. Gur, "Digital breast tomosynthesis in the diagnostic environment: a subjective side-by-side review," American Journal of Roentgenology, vol. 195, no. 2, pp. W172-W176, 2010.

[33] K. R. Brandt, D. A. Craig, T. L. Hoskins et al., "Can digital breast tomosynthesis replace conventional diagnostic mammography views for screening recalls without calcifications? A comparison study in a simulated clinical setting," American Journal of Roentgenology, vol. 200, no. 2, pp. 291-298, 2013.

[34] R. J. Hooley, K. L. Greenberg, R. M. Stackhouse, J. L. Geisel, R. S. Butler, and L. E. Philpotts, "Screening US in patients with mammographically dense breasts: initial experience with Connecticut public act 09-41," Radiology, vol. 265, no. 1, pp. 59-69, 2012.

[35] F. M. Hall, R. J. Hooley, K. Greenberg et al., "Breast density legislation,” Radiology, vol. 266, no. 3, pp. 997-998, 2013.

[36] I. J. Hall, A. Middlebrooks, and S. S. Coughlin, "Population prevalence of first-degree family history of breast and ovarian cancer in the United States: implications for genetic testing," The Open Health Services and Policy Journal, vol. 1, pp. 34-37, 2008. 


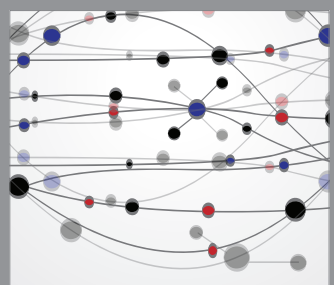

The Scientific World Journal
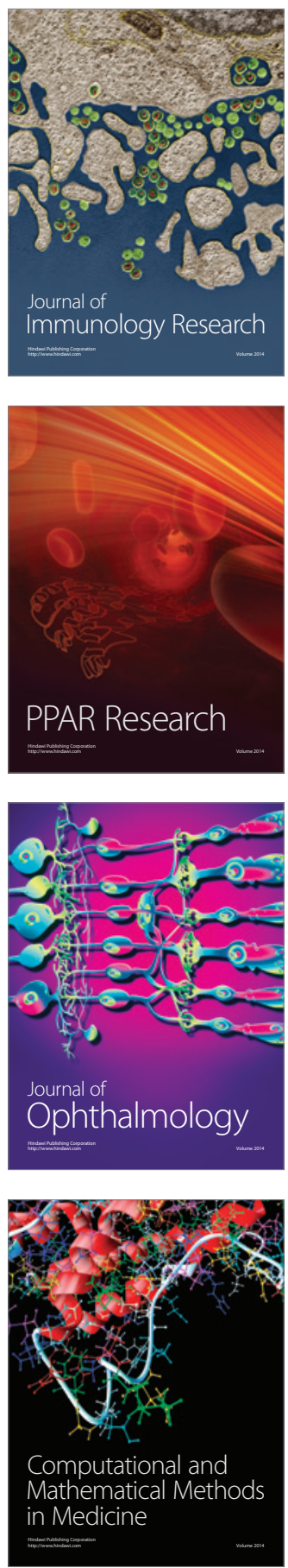

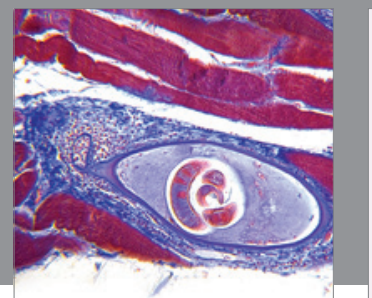

Gastroenterology

Research and Practice
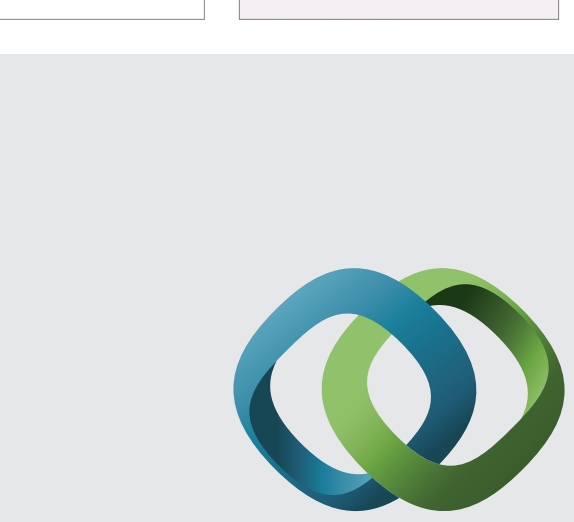

\section{Hindawi}

Submit your manuscripts at

http://www.hindawi.com
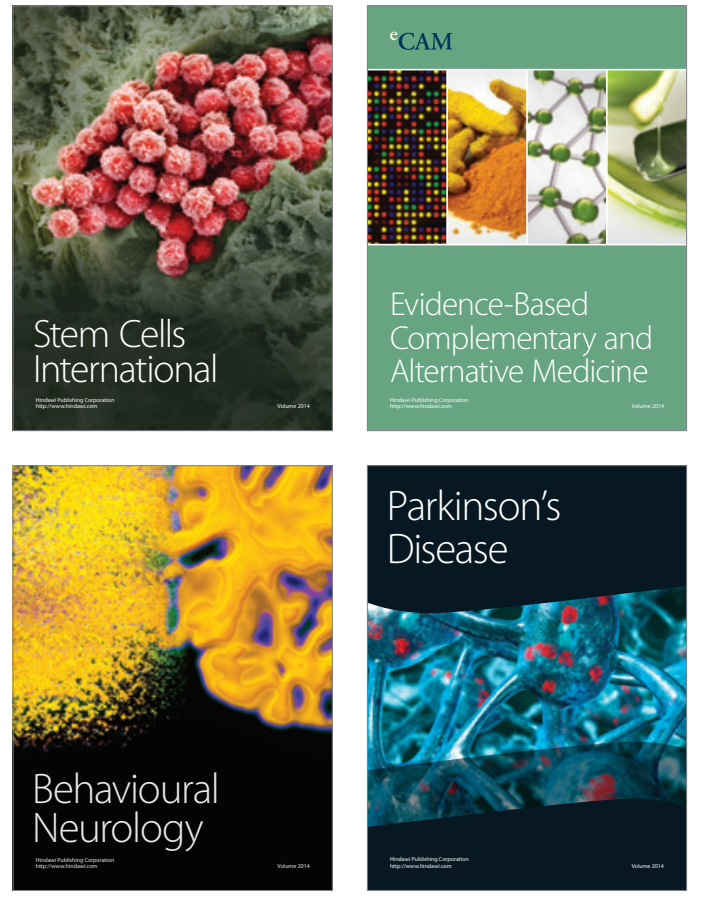
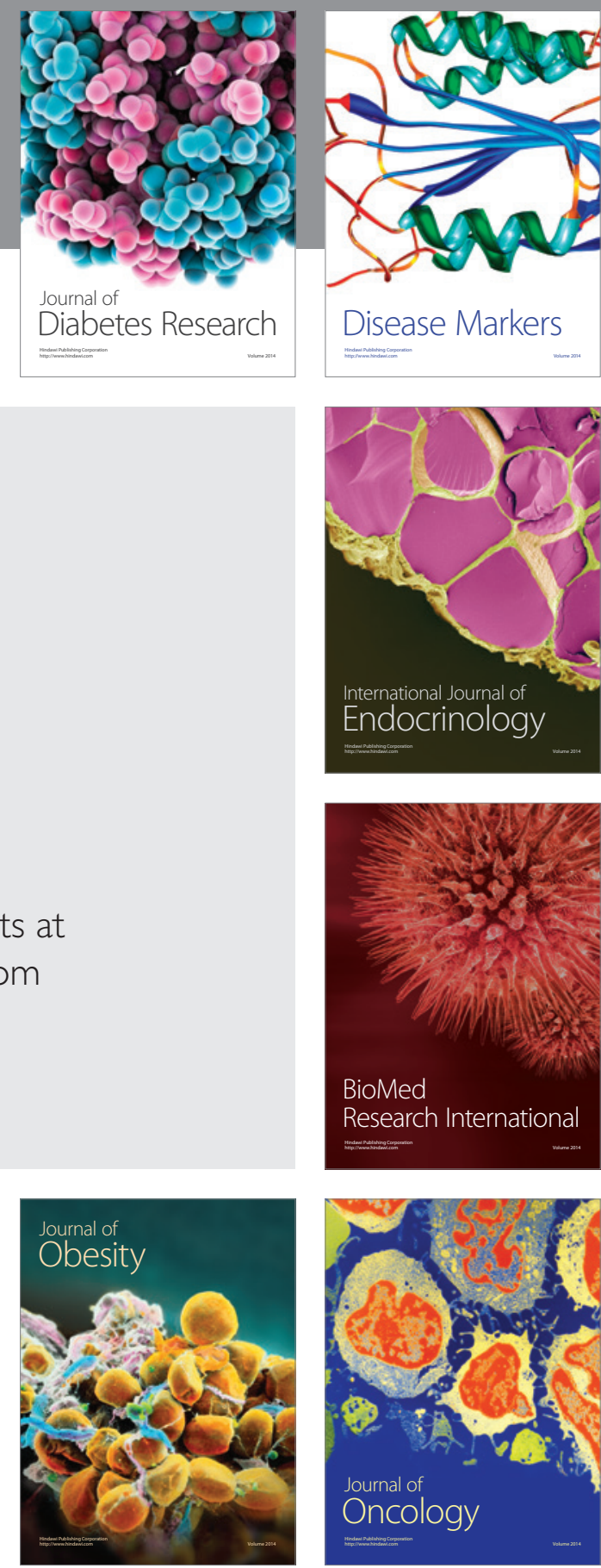

Disease Markers
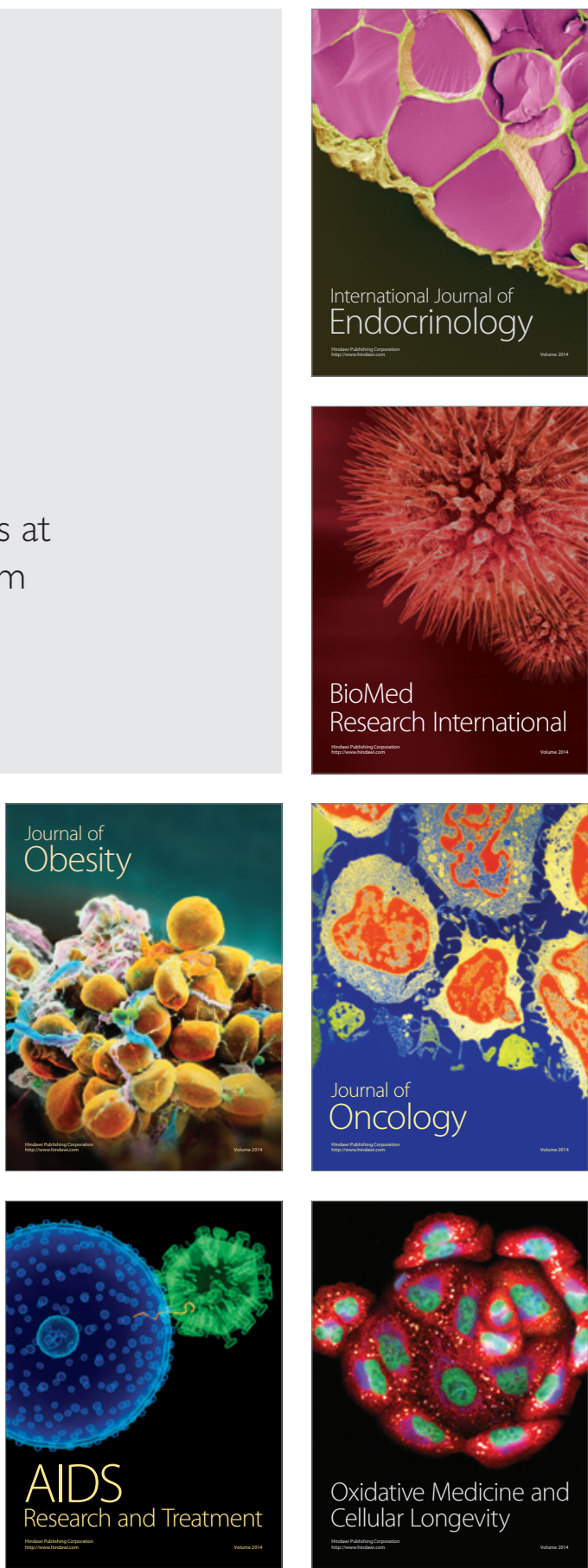